\title{
Fibrewise Compactly-Generated Spaces
}

Ioan M. JAMES*

In homotopy theory it is convenient to work with a class of spaces which is closed under the standard operations of the theory and yet is large enough to contain all the spaces which are usually considered interesting. The compactly-generated spaces of Steenrod [9] form such a class. With Steenrod these spaces are required to satisfy the Hausdorff separation condition and this leads to difficulties when it is necessary to form quotients. However McCord [7] showed that these difficulties could be overcome, without losing other desirable properties, if a weaker separation condition was used instead. It should be mentioned, before going any further, that R. Brown [4] had earlier developed a similar theory to Steenrod's, that Booth [1], Spanier, Vogt and others have also presented related theories, furthermore that McCord ascribes the idea for his variant of the Steenrod theory to J. C. Moore, while Booth ascribes a similar idea to A. Clark.

In fibrewise homotopy theory there is a similar need to define a sufficiently extensive class of fibrewise spaces which is closed under the standard operations of the theory. A fibrewise generalization of Steenrod's class of compactlygenerated spaces is developed, up to a certain stage, in $\S 13$ of [6]. Although these fibrewise compactly-generated spaces have many of the desired properties the fibrewise Hausdorff requirement leads to serious difficulties beyond that stage. However, as we shall see here, these difficulties can be avoided, and a much more satisfactory theory obtained, if Steenrod's theory is replaced by McCord's. What follows, therefore, is a fibrewise generalization of the McCord version of the Steenrod theory. Some applications will be given in a sequel. Fibrewise versions of the other theories mentioned in the previous paragraph either have been developed (see Booth and Brown [2], for example) or could be developed.

For the basic notions of fibrewise topology I refer the reader to [6], where all the results we shall be using may be found. The systematic terminol-

Communicated by K. Saito, December 27, 1993.

1991 Mathematics Subject Classifications: 54C35, 55R99.

* Mathematical Institute, University of Oxford, 24-29 St. Giles, Oxford OX1 3LB, GREAT BRITAIN. 
ogy used in [6], which I hope is largely self-explanatory, will be adopted here, except that it is convenient to follow the usage of Bourbaki and include the fibrewise Hausdorff condition in the definition of the terms fibrewise compact and fibrewise locally compact.

\section{$\S 1$. The Retraction Functor}

We work over an arbitrary base space, usually denoted by $B$. We need a fibrewise version of the weak Hausdorff condition, as follows.

Definition (1.1). The fibrewise space $X$ over $B$ is fibrewise weak Hausdorff if for each open set $W$ of $B$, each fibrewise compact space $K$ over $W$, and each fibrewise map $\alpha: K \rightarrow X_{W}$, the image $\alpha K$ is closed in $X_{W}$.

For example, sections of fibrewise weak Hausdorff spaces are closed. Clearly fibrewise Hausdorff implies fibrewise weak Hausdorff; it is easy to give examples where the reverse implication is false. Subspaces of fibrewise weak Hausdorff spaces are fibrewise weak Hausdorff, as are fibrewise products of fibrewise weak Hausdorff spaces. Restrictions to open subspaces of fibrewise weak Hausdorff spaces are fibrewise weak Hausdorff.

Let $X$ be a fibrewise space over $B$. We describe a subset $H$ of $X$ as fibrewise-compactly-closed if for each open set $W$ of $B$, each fibrewise compact space $K$ over $W$, and each fibrewise map $\alpha: K \rightarrow X_{W}$, the preimage $\alpha^{-1}\left(X_{W} \cap H\right)$ is closed in $K$. This is the case, of course, when $H$ is closed in $X$. We describe $X$ as a $k_{B}$-space if every fibrewise-compactly-closed subset is closed.

Definition (1.2). The fibrewise space $X$ over $B$ is fibrewise compactlygenerated if (i) $X$ is fibrewise weak Hausdorff and (ii) $X$ is a $k_{B}$-space.

Restrictions of fibrewise compactly-generated spaces to closed subspaces are also fibrewise compactly-generated.

Proposition (1.3). Let $X$ be a fibrewise locally compact space over $B$. Then $X$ is fibrewise compactly-generated.

Since fibrewise locally compact, in this paper, includes fibrewise Hausdorff, we only need to prove (ii). So let $H$ be a fibrewise-compactly-closed subset of $X$ and let $x \notin H$ where $x \in X_{b}, b \in B$. Since $X$ is fibrewise locally compact there exists a neighbourhood $W$ of $b$ in $B$ and a neighbourhood $N$ of $x$ in $X_{W}$ such that the closure $X_{W} \cap \bar{N}$ of $N$ in $X_{W}$ is fibrewise compact over $W$. Since $H$ is fibrewise-compactly-closed the intersection $X_{W} \cap H \cap \bar{N}$ is closed in $X_{W} \cap \bar{N}$ and so $\left(X_{W}-X_{W} \cap H\right) \cap \bar{N}$ is open in $X_{W} \cap \bar{N}$. Taking intersections with $N$ we obtain that $\left(X_{W}-X_{W} \cap H\right) \cap N$ is open in $X_{W} \cap N$ and so open in $X$. Thus $x$ admits a neighbourhood in $X$ which does not meet $H$. Therefore $H$ is closed. We conclude that $X$ is a $k_{B}$-space and so fibrewise compactlygenerated, as asserted. 
Proposition (1.4). Let $X$ be fibrewise compactly-generated over $B$. Let $Y$ be a fibrewise weak Hausdorff fibrewise quotient of $X$. Then $Y$ is fibrewise compactly-generated.

For consider the natural projection $\pi: X \rightarrow Y$. Let $H$ be fibrewise-compactly-closed in $Y$. I assert that the preimage $\pi^{-1} H$ is fibrewise-compactlyclosed in $X$, therefore closed in $X$, and so $H$ is closed in $Y$. For let $\alpha: K \rightarrow X_{W}$ be a fibrewise map, where $W$ is open in $B$ and $K$ is fibrewise compact over $W$. Since $H$ is fibrewise-compactly-closed in $Y$ we have that $\alpha^{-1} \pi^{-1} H=(\pi \alpha)^{-1} H$ is closed in $K$. Thus $\pi^{-1} H$ is fibrewise-compactly-closed in $X$, as asserted.

Turning now to questions of continuity we find that another auxiliary concept is of value. Let $\phi: X \rightarrow Y$ be a fibrewise function, where $X$ and $Y$ are fibrewise spaces over $B$. Let us describe $\phi$ as quasi-continuous if for each open set $W$ of $B$, each fibrewise compact space $K$ over $W$, and each fibrewise map $\alpha: K \rightarrow X_{W}$, the composition $\phi \circ \alpha: K \rightarrow Y_{W}$ is continuous. This is the case, of course, when $\phi$ is continuous. In the other direction we have

Proposition (1.5). Let $X$ and $Y$ be fibrewise spaces over $B$, with $X$ fibrewise compactly-generated and $Y$ fibrewise weak Hausdorff. Let $\phi: X \rightarrow Y$ be a quasicontinuous fibrewise function. Then $\phi$ is continuous.

For let $H$ be closed in $Y$. I assert that the preimage $\phi^{-1} H$ is fibrewisecompactly-closed in $X$ and so closed in $X$, since $X$ is fibrewise compactlygenerated. For let $\alpha: K \rightarrow X_{W}$ be a fibrewise map, where $W$ is open in $B$ and $K$ is fibrewise compact over $W$. Then $\phi \circ \alpha: K \rightarrow Y_{W}$ is continuous, since $\phi$ is quasi-continuous, and so $\phi \alpha K$ is closed in $Y_{W}$, since $Y$ is fibrewise weak Hausdorff. Hence $H \cap \phi \alpha K$ is closed in $Y_{W}$, and so

$$
(\phi \alpha)^{-1}(H \cap \phi \alpha K)=(\phi \alpha)^{-1} H \cap K
$$

is closed in $K$. Therefore $\phi^{-1} H$ is fibrewise-compactly-closed, as asserted.

We are now ready to define a functor $k_{B}$ from the category of fibrewise weak Hausdorff spaces over $B$ to the category of fibrewise compactly-generated spaces over $B$, as follows. Given a fibrewise weak Hausdorff space $X$ over $B$ we put $k_{B} X=X^{\prime}$, the same fibrewise set as $X$ but with the refinement of the fibrewise topology in which fibrewise-compactly-closed sets are closed. Given a quasi-continuous fibrewise function $\phi: X \rightarrow Y$, where $X$ and $Y$ are fibrewise weak Hausdorff over $B$, we put $k_{B} \phi=\phi^{\prime}$, the same fibrewise function but with $\phi^{\prime}: X^{\prime} \rightarrow Y^{\prime}$, so that $\phi^{\prime}$ is continuous. It is easy to see that $k_{B} X$ is fibrewise compactly-generated, for all fibrewise weak Hausdorff $X$, and that $k_{B} X=X$ when $X$ is fibrewise compactly-generated. We shall therefore refer to $k_{B}$ as the retraction functor.

It should be noted that the retraction functor commutes with the restriction functor, provided restriction is to open subsets of the base. Specifically 
let $X$ be a fibrewise weak Hausdorff space over $B$, and let $A$ be an open subspace of $B$. Then the restriction of $k_{B} X$ to $A$ coincides with $k_{A} X_{A}$.

\section{§ 2. The Fibrewise Compactly-Generated Fibrewise Product}

Given fibrewise compactly-generated spaces $X, Y$ over $B$ we define their fibrewise compactly-generated fibrewise product to be the result of applying the functor $k_{B}$ to the ordinary fibrewise product $X \times_{B} Y$. The notation $X \times_{B}^{\prime} Y=k_{B}\left(X \times_{B} Y\right)$ is convenient. It is easy to see that the fibrewise compactly-generated fibrewise product has the necessary formal properties. First of all the projections into the factors are continuous, since the identity function $X \times{ }_{B}^{\prime} Y \rightarrow X \times{ }_{B} Y$ is continuous. To establish the cartesian property, let

$$
X \stackrel{\phi}{\leftarrow} A \stackrel{\psi}{\rightarrow} Y
$$

be a cotriad of fibrewise maps, where $A$ is fibrewise compactly-generated. Then $\phi$ and $\psi$ are components of a unique fibrewise map $\theta: A \rightarrow X \times_{B} Y$. Applying the functor $k_{B}$ and using the fact that $A^{\prime}=A$ we obtain the unique fibrewise map $A \rightarrow X \times_{B}^{\prime} Y$ which, when composed with the projections, gives $\phi$ and $\psi$. From this result it follows that the fibrewise compactly-generated fibrewise product satisfies the usual laws of commutativity and associativity. The construction can be extended to any number of factors in a similar fashion.

Proposition (2.1). Let $X$ and $Y$ be fibrewise spaces over $B$, with $X$ fibrewise locally compact and $Y$ locally sliceable. Suppose that $Y$ is fibrewise compactlygenerated. Then $X \times_{B} Y$ is fibrewise compactly-generated, and so

$$
X \times_{B}^{\prime} Y=X \times_{B} Y \text {. }
$$

For let $H$ be a fibrewise-compactly-closed subset of $X \times_{B} Y$. Let $(x, y) \notin H$, where $x \in X_{b}, y \in Y_{b}, b \in B$. Since $X$ is fibrewise locally compact there exists a neighbourhood $W$ of $b$ in $B$ and a neighbourhood $N$ of $x$ in $X_{W}$ such that the closure $X_{W} \cap \bar{N}$ of $N$ in $X_{W}$ is fibrewise compact over $W$. By shrinking $W$, if necessary, we can arrange for $Y$ to admit a section $t$ over $W$ such that $t(b)=y$. Then $H$ meets $\left(X_{W} \cap \bar{N}\right) \times_{W} t W$ in a closed set, since $\left(X_{W} \cap \bar{N}\right) \times_{W} t W$ is fibrewise compact over $W$. Since the intersection is closed in $X_{W} \times_{W} t W$ it follows that $x$ admits a neighbourhood $U \subset N$ such that $\left(X_{W} \cap \bar{U}\right) \times_{W} t W$ does not meet $H$. Let $H_{Y}$ denote the second projection of

$$
H \cap\left(\left(X_{W} \cap \bar{U}\right) \times_{W} t W\right),
$$

so that $H_{Y} \subset Y_{W}$. I assert that $H_{Y}$ is fibrewise-compactly-closed in $Y_{W}$, hence closed in $Y_{W}$, since $Y$ is fibrewise compactly-generated. 
For let $\alpha: K \rightarrow Y_{V}$ be a fibrewise map, where $V$ is open in $W$ and $K$ is fibrewise compact over $V$. The fibrewise product

$$
\beta:\left(X_{V} \cap \bar{U}\right) \times_{V} K \rightarrow X_{V} \times{ }_{V} Y_{V}
$$

of the inclusion and $\alpha$ is a fibrewise map. Since $\left(X_{V} \cap \bar{U}\right) \times_{V} K$ is fibrewise compact over $V$, and $H$ is fibrewise-compactly-closed, we obtain that $\beta^{-1} H$ is closed and so $\alpha^{-1} H_{Y}$ is closed, as required. Now $y \notin H_{Y}$ and so $\left(U_{V} \cap X_{V}\right) \times_{V}$ $\left(Y_{V}-H_{Y}\right)$ is a neighbourhood of $(x, y)$ which does not meet $H$. Therefore $H$ is closed, which proves (2.1).

Proposition (2.2). Let $\pi: X \rightarrow Y$ be a fibrewise quotient map, where $X$ and $Y$ are fibrewise compactly-generated over $B$, with $Y$ locally sliceable. Then the fibrewise product

$$
\pi \times i d: X \times \times_{B}^{\prime} T \rightarrow Y \times{ }_{B}^{\prime} T
$$

is also a fibrewise quotient map, for all fibrewise compactly-generated $T$.

For let $H$ be a subset of $Y \times_{B}^{\prime} T$ such that the preimage $(\pi \times i d)^{-1} H$ is closed in $X \times_{B}^{\prime} T$. I assert that $H$ is fibrewise-compactly-closed and so closed in $Y \times_{B}^{\prime} T$, since $Y \times_{B}^{\prime} T$ is fibrewise compactly-generated. For let $\beta: K \rightarrow Y_{W}$ $\times_{W}^{\prime} T_{W}$ be a fibrewise map, where $W$ is open in $B$ and $K$ is fibrewise compact over $W$. Consider the fibrewise map

$$
\beta_{1} \times \beta_{2}: K \times_{W}^{\prime} K \rightarrow Y_{W} \times_{W}^{\prime} T_{W},
$$

where $\beta_{1}, \beta_{2}$ are the components of $\beta$. Now $K \times_{W}^{\prime} K=K \times_{W} K$, by (2.1), which is fibrewise compact over $W$. I assert that $\left(\beta_{1} \times \beta_{2}\right)^{-1} H$ is closed in $K \times{ }_{W} K$, hence $\beta^{-1} H$ is closed in $K$.

For consider the fibrewise product $X_{W} \times_{Y_{W}} K$, where $X_{W}$ is regarded as a fibrewise space over $Y_{W}$ using $\pi$ while $K$ is regarded as a fibrewise space over $Y_{W}$ using $\beta_{1}$. Now $K$ is fibrewise compact over $Y_{W}$, by (3.19) of [6], since $K$ is fibrewise compact over $W$, and $Y_{W}$ is fibrewise Hausdorff over $W$. Hence

$$
\pi_{2}: X_{W} \times_{Y_{W}} K \rightarrow K
$$

is a fibrewise quotient map, and hence

$$
\pi_{2} \times i d: X_{W} \times{ }_{Y_{W}} K \times_{W} K \rightarrow K \times_{W} K
$$

is a fibrewise quotient. Consider the fibrewise product

$$
\pi_{1} \times \beta_{2}: X_{W} \times_{Y_{W}} K \times_{W} K \rightarrow X_{W} \times_{W} T_{W}
$$

of the first projection and $\beta_{2}$. Since $(\pi \times i d)^{-1} H$ is closed in $X \times_{B} T$ it follows that

$$
\left(\pi_{1} \times \beta_{2}\right)^{-1}(\pi \times i d)^{-1} H=\left(\pi_{2} \times i d\right)^{-1}\left(\beta_{1} \times \beta_{2}\right)^{-1} H
$$


is closed in $X_{W} \times_{Y_{W}} K \times_{W} K$, and so $\left(\beta_{1} \times \beta_{2}\right)^{-1} H$ is closed in $K \times_{W} K$, as asserted. Thus $\beta^{-1} H$ is closed in $K$. This shows that $H$ is fibrewise-compactlyclosed and so $H$ is closed, since $Y \times_{B}^{\prime} T$ is fibrewise compactly-generated. Therefore $\pi \times$ id is a fibrewise quotient map, as asserted.

Proposition (2.3). Let $X$ and $Y$ be fibrewise weak Hausdorff spaces over B. Then

$$
X^{\prime} \times_{B}^{\prime} Y^{\prime}=X \times_{B}^{\prime} Y
$$

To see this first observe that the identity function $u: X^{\prime} \times_{B} Y^{\prime} \rightarrow X \times_{B} Y$ is continuous, since the identity functions $X^{\prime} \rightarrow X$ and $Y^{\prime} \rightarrow Y$ are continuous. Let $W$ be open in $B$ and let $K$ be fibrewise compact over $B$. Each fibrewise map $\alpha^{\prime}: K \rightarrow X_{W}^{\prime} \times_{W} Y_{W}^{\prime}$ determines a fibrewise map $\alpha=u \circ \alpha^{\prime}: K \rightarrow$ $X_{W} \times_{W} Y_{W}$. On the other hand let $\alpha: K \rightarrow X_{W} \times_{W} Y_{W}$ be a fibrewise map, with projections

$$
X_{W} \stackrel{\alpha_{1}}{\leftarrow} K \stackrel{\alpha_{2}}{\rightarrow} Y_{W}
$$

Then $\alpha_{1} \times \alpha_{2}: K \times_{W} K \rightarrow X_{W}^{\prime} \times_{W} Y_{W}^{\prime}$ is continuous, hence the fibrewise function $\alpha^{\prime}: K \rightarrow X_{W}^{\prime} \times_{W} Y_{W}^{\prime}$ is continuous, where $u \circ \alpha^{\prime}=\alpha$. Hence the associated fibrewise compactly-generated topologies coincide.

The following characterization of the fibrewise weak Hausdorff property, for the class of $k_{B}$-spaces, is a fibrewise version of (2.3) of [7].

Proposition (2.4). The $k_{B}$-space $X$ over $B$ is fibrewise weak Hausdorff if and only if the diagonal $\Delta X$ is closed in $X \times_{B}^{\prime} X$.

For suppose that $\Delta X$ is closed in $X \times_{B}^{\prime} X$. Let $\alpha: K \rightarrow X_{W}$ be a fibrewise map, where $W$ is open in $B$ and $K$ is fibrewise compact over $W$. I assert that $\alpha K$ is fibrewise-compactly-closed in $X_{W}$ and hence closed. To see this let $b$ be a point of $W$, let $V$ be a neighbourhood of $b$ in $W$, let $L$ be fibrewise compact over $V$ and let $\beta: L \rightarrow X_{V}$ be a fibrewise map. Then $K_{V} \times_{V} L$ is fibrewise compact over $V$, and

$$
\alpha \times \beta: K_{V} \times_{V}^{\prime} L \rightarrow X_{V} \times_{V}^{\prime} X_{V}
$$

is a fibrewise map. Since $\Delta X$ is closed in $X \times_{B}^{\prime} X$, hence fibrewise-compactlyclosed in $X \times{ }_{B} X$, we have that $(\alpha \times \beta)^{-1} \Delta X_{V}$ is closed in $K_{V} \times_{V}^{\prime} L$. Hence $\beta^{-1}\left(\alpha K_{V}\right)=\pi_{2}(\alpha \times \beta)^{-1} \Delta X_{V}$ is closed in $L_{V}$. Thus $\alpha K$ is closed in $X_{W}$, therefore $X$ is fibrewise weak Hausdorff.

Conversely suppose that $X$ is fibrewise weak Hausdorff. I assert that $\Delta X$ is closed in $X \times_{B}^{\prime} X$. For let $\gamma: K \rightarrow X_{W} \times_{W}^{\prime} X_{W}$ be a fibrewise map, where $W$ is open in $B$ and $K$ is fibrewise compact over $W$. Then

$$
A=\left(\pi_{1} \gamma K\right) \cup\left(\pi_{2} \gamma K\right)
$$


is closed in $X_{W}$, since $X$ is fibrewise weak Hausdorff. Now $\triangle A$ is closed in $A \times_{W}^{\prime} A$, since $\gamma K$ is fibrewise Hausdorff, and so $\gamma^{-1} \Delta X=\gamma^{-1} \Delta A$ is closed in $K$. Thus $\Delta X$ is fibrewise-compactly-closed, and so closed in the fibrewise compactly-generated $X \times{ }_{B}^{\prime} X$.

We obtain the useful

Proposition (2.5). Let $X$ and $Y$ be fibrewise spaces over $B$, with $X$ fibrewise compactly-generated. Let $\pi: X \rightarrow Y$ be a fibrewise quotient map. Then $Y$ is fibrewise compactly-generated if and only if $(\pi \times \pi)^{-1} \Delta Y$ is closed in $X \times \times_{B}^{\prime} X$.

Corollary (2.6). Let $X$ and $Y$ be fibrewise spaces over $B$, with $X$ fibrewise compactly-generated. Let $h:\left(X, X_{0}\right) \rightarrow\left(Y, Y_{0}\right)$ be a fibrewise relative homeomorphism where $X_{0} \subset X$ and $Y_{0} \subset Y$. If $Y_{0}$ is fibrewise compactly-generated then so is $Y$. In particular the fibrewise collapse $X /{ }_{B} X_{0}$ is fibrewise compactlygenerated.

The proposition follows at once from (2.4), and to deduce the corollary we simply observe that

$$
(h \times h)^{-1} \Delta Y=\Delta X \cup(h \times h)^{-1} \Delta Y_{0} .
$$

So far the exposition has run more or less parallel to that given in $\S 13$ of [6] although a weaker fibrewise separation condition is being used in the present account and the meaning of the term fibrewise compactly-generated has been tightened up somewhat. Beyond this stage it is difficult to proceed any further with the earlier version but in the present theory the same difficulties do not arise and the exposition can be continued as follows.

\section{§3. The Fibrewise Pointed Theory}

We turn now to the category of fibrewise pointed spaces, i.e. fibrewise spaces with closed section. Given a fibrewise weak Hausdorff fibrewise pointed space $X$ with closed section $s: B \rightarrow X$ we regard $X^{\prime}$ as a fibrewise pointed space with section $s^{\prime}: B \rightarrow X^{\prime}$, which is also closed.

Let $X$ be a fibrewise space over $B$ and let $A$ be a closed subset of $X$. Then $A$ is also a closed subset of $X^{\prime}$ and so the fibrewise collapse $X^{\prime} /{ }_{B} A$ is fibrewise compactly-generated.

For example let $X$ and $Y$ be fibrewise pointed spaces over $B$. The fibrewise wedge-product $X \vee_{B} Y$ is defined, in the usual way, as a fibrewise pointed subset of $X \times_{B} Y$, and we denote by $X \vee_{B}^{\prime} Y$ the corresponding subset of $X \times_{B}^{\prime} Y$, with the relative topology. Moreover we denote by $X \wedge_{B}^{\prime} Y$ the fibrewise pointed space obtained from $X \times_{B}^{\prime} Y$ by fibrewise collapsing the closed subset $X \vee_{B}^{\prime} Y$. Both $X \vee_{B}^{\prime} Y$ and $X \wedge_{B}^{\prime} Y$ are fibrewise compactly-generated. 
Note that if $X$ and $Y$ are fibrewise weak Hausdorff then

$$
X \vee_{B}^{\prime} Y=X^{\prime} \vee_{B}^{\prime} Y^{\prime}, \quad X \wedge_{B}^{\prime} Y=X^{\prime} \wedge_{B}^{\prime} Y^{\prime},
$$

by (2.3). Also that if $X$ is fibrewise locally compact and if $Y$ is both locally sliceable and fibrewise compactly-generated then

$$
X \vee_{B}^{\prime} Y=X \vee_{B} Y, \quad X \wedge_{B}^{\prime} Y=X \wedge_{B} Y,
$$

by (2.3).

Proposition (3.1). Let $X, Y$ and $Z$ be fibrewise compactly-generated fibrewise pointed spaces over $B$. Then there exists a natural equivalence

$$
\left(X \wedge_{B}^{\prime} Z\right) \vee_{B}^{\prime}\left(Y \wedge_{B}^{\prime} Z\right) \rightarrow\left(X \vee_{B}^{\prime} Y\right) \wedge_{B}^{\prime} Z
$$

of fibrewise pointed spaces.

For consider the diagram shown below, where the arrows represent the obvious functions.

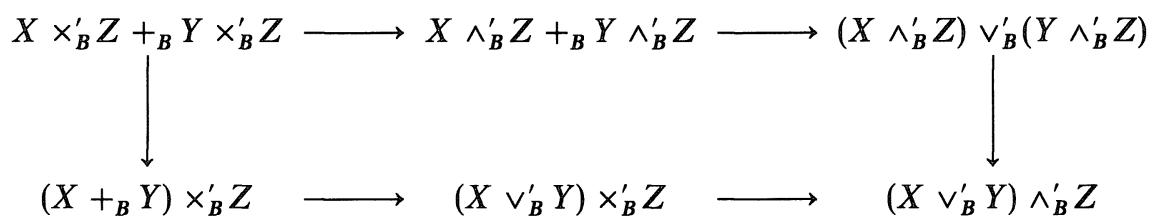

The horizontals on the right are fibrewise quotients by definition. The upper horizontal on the left is a fibrewise coproduct of fibrewise quotients, therefore a fibrewise quotient. The lower horizontal on the left is the fibrewise product of the fibrewise quotient with the identity, and so also a fibrewise quotient. The left-hand vertical is a fibrewise topological equivalence by the distributive law for the fibrewise product. The right-hand vertical is a bijection and hence, by commutativity of the diagram, a fibrewise topological equivalence.

Proposition (3.2). Let $X, Y$ and $Z$ be fibrewise compactly-generated fibrewise pointed spaces over $B$. Then there exists a natural equivalence

$$
\left(X \wedge_{B}^{\prime} Y\right) \wedge_{B}^{\prime} Z \rightarrow X \wedge_{B}^{\prime}\left(Y \wedge_{B}^{\prime} Z\right)
$$

of fibrewise pointed spaces.

To prove this consider the diagram shown below, where the horizontal arrows represent the obvious functions and the right-hand vertical is induced by the identity at the left.

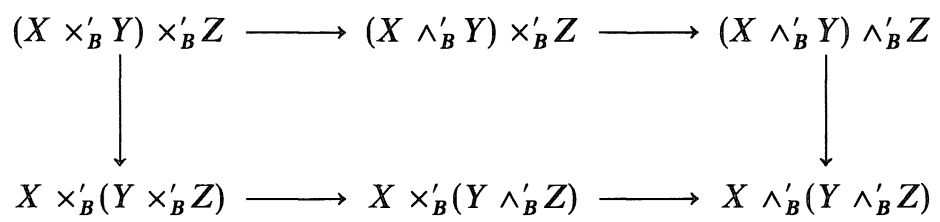


Since all the other arrows in the diagram represent fibrewise quotients it follows that the arrow on the right is an equivalence of fibrewise pointed spaces, as required.

We refer to the last two results as the distributive law and the associative law for the fibrewise smash product. The proof of our next result is similar and will therefore be omitted.

Proposition (3.3). Let $X$ and $Y$ be fibrewise pointed spaces over $B$, and let $A$ be a closed subspace of $X$. Suppose that $X$ and $Y$ are fibrewise compactlygenerated. Then there exists a natural equivalence

$$
\left(X \wedge_{B}^{\prime} Y\right) /{ }_{B}\left(A \wedge_{B}^{\prime} Y\right) \rightarrow\left(X /{ }_{B} A\right) \wedge_{B}^{\prime} Y
$$

of fibrewise pointed spaces.

\section{§4. The Fibrewise Compact-Open Topology}

I have previously discussed fibrewise mapping spaces in $\S 9$ of [6], using a fibrewise version of the compact-open topology. In what follows I slightly modify the definition used in [6], without significantly affecting the properties of the functor, and this modification will enable us to complete our discussion of fibrewise compactly-generated spaces, in the remaining two sections. I should mention that the problem of constructing an explicit right adjoint to the fibrewise product has been considered elsewhere in the literature, using various methods. For example Booth and Brown [2], [3] use partial maps while Min and Lee [8] use convergence spaces.

Let us begin with a slight modification of the standard compact-open topology. Given spaces $X$ and $Y$ let map $(X, Y)$ denote the set of maps $X \rightarrow Y$. For $U \subset Y$ open, $C$ compact and $\alpha: C \rightarrow X$ a map, let $(C, \alpha, U)$ denote the subset of map $(X, Y)$ consisting of maps $\phi$ such that $\phi \alpha C \subset U$. We describe such sets as compact-open and we describe the topology which they generate as the compact-open topology. In the standard theory $C$ is required to be a subset of $X$ and $\alpha$ the inclusion, but our refinement of the standard topology has all the expected properties, as can easily be checked.

Turning now to the fibrewise theory, we need to assign a suitable fibrewise topology to the fibrewise set

$$
\operatorname{map}_{B}(X, Y)=\coprod_{b \in B} \operatorname{map}\left(X_{b}, Y_{b}\right),
$$

where $X$ and $Y$ are fibrewise spaces over $B$. For this we need a fibrewise version of the compact-open topology. A fibrewise version of the standard compact-open topology has been given in $\S 9$ of [6]. A fibrewise version of the modified compact-open topology is as follows. Given an open set $W \subset B$, an open set $U \subset Y_{W}$, a fibrewise compact space $K$ over $W$, and a fibrewise 
$\operatorname{map} \alpha: K \rightarrow X_{W}$, we denote by $(K, \alpha, U ; W)$ the set of maps $\phi: X_{b} \rightarrow Y_{b}$, where $b \in$ $W$, for which $\phi \alpha K_{b} \subset U$. We describe such subsets $(K, \alpha, U ; W)$ of $\operatorname{map}_{B}(X, Y)$ as fibrewise compact-open, and describe the fibrewise topology which they generate as the fibrewise compact-open topology. In case $K \subset X_{W}$ and $\alpha$ is the inclusion we write $(K, \alpha, U ; W)$ as $(K, U ; W)$. In $\S 9$ of [6] the fibrewise compact-open subsets are all of this form, but the modification we are making here does not affect the properties of the construction in any significant way.

For example, it is shown in $\S 9$ of [6] that if $X$ is locally sliceable and $Y$ is fibrewise Hausdorff then $\operatorname{map}_{B}(X, Y)$ is fibrewise Hausdorff. This remains true after the modification in the fibrewise compact-open topology. However due to the nature of the modification it is the fibrewise weak Hausdorff property (1.1) which is more relevant now, and so we prove

Proposition (4.1). Let $X$ and $Y$ be fibrewise spaces over $B$, with $X$ locally sliceable. If $Y$ is fibrewise weak Hausdorff then so is $\operatorname{map}_{B}(X, Y)$.

For let $W \subset B$ be open, let $K$ be fibrewise compact over $W$, and let

$$
\alpha:\left.K \rightarrow \operatorname{map}_{B}(X, Y)\right|_{W}
$$

be a fibrewise map. Let $\phi: X_{b} \rightarrow Y_{b}$, where $b \in W$, lie outside $\alpha K$. Then there exists a point $x$ of $X_{b}$ such that $\phi(x) \neq \alpha(\xi)(x)$ for all $\xi \in K_{b}$. Let $V \subset W$ be a neighbourhood of $b$ for which there exists a slice $s: V \rightarrow X_{V}$ with $s(b)=x$. By composing $\alpha$ with $s^{*}$ and then restricting we obtain a fibrewise map $\beta: K_{V} \rightarrow Y_{V}$. Assume that $Y$ is fibrewise weak Hausdorff, and so $\beta K_{V}$ is closed in $Y_{V}$. Since $\phi(x) \notin K_{V}$ there exists a neighbourhood $N$ of $\phi(x)$ in $Y_{V}$ which does not meet $\beta K_{V}$. Then $(V, s, N ; V)$ is a fibrewise compact-open neighbourhood of $\phi$ in $\operatorname{map}_{B}(X, Y)$ which does not meet $\alpha K_{V}$ and hence does not meet $\alpha K$. Since $V \subset W$ this shows that $\operatorname{map}_{B}(X, Y)$ is fibrewise weak Hausdorff.

\section{§5. Fibrewise Compactly-Generated Mapping-Spaces}

The fibrewise compact-open topology has many desirable properties but some of them, such as the exponential law, are subject to inconvenient restrictions. In the case of the ordinary theory Steenrod [9] has shown these restrictions can be avoided by applying the retraction functor, and this is true whether his original version is used or McCord's variant. As we shall now see, similar results hold in the fibrewise theory.

We continue to work over a base space $B$, without restriction. If $X$ and $Y$ are fibrewise spaces over $B$ we denote by $\operatorname{map}_{B}(X, Y)$ the fibrewise mappingspace, with fibrewise compact-open topology. Suppose that $X$ is locally sliceable. Then $\operatorname{map}_{B}(X, Y)$ is fibrewise weak Hausdorff for all fibrewise weak Hausdorff $Y$, by (4.1). Under these conditions the fibrewise compactly-generated fibrewise 
mapping-space

$$
\operatorname{map}_{B}^{\prime}(X, Y)=k_{B} \operatorname{map}_{B}(X, Y)
$$

is defined.

Lemma (5.1). Let $X$ and $Y$ be fibrewise spaces over $B$. Let $W \subset B$ be open, let $K$ be fibrewise compact over $W$, and let

$$
\alpha: K \rightarrow \operatorname{map}_{W}\left(X_{W}, Y_{W}\right) \times_{W} Y_{W}
$$

be a fibrewise map. Then each point $b$ of $W$ admits a neighbourhood $V \subset W$ such that $e \circ \alpha: K_{V} \rightarrow Y_{V}$ is continuous, where $e$ denotes the fibrewise evaluation function.

Clearly it is sufficient to show that for each pair of fibrewise maps

$$
\alpha_{1}: K \rightarrow \operatorname{map}_{W}\left(X_{W}, Y_{W}\right), \quad \alpha_{2}: K \rightarrow Y_{W}
$$

each point $b$ of $W$ admits a neighbourhood $V \subset W$ such that $e \circ\left(\alpha_{1} \times \alpha_{2}\right)$ : $K_{V} \times_{V} K_{V} \rightarrow Y_{V}$ is continuous. So let $\xi, \eta \in K_{b}$ and let $U$ be a neighbourhood of $\beta \alpha_{1}(\xi)\left(\alpha_{2}(\xi)\right)$ in $Y_{W}$. Since $\alpha_{1}(\xi): X_{b} \rightarrow Y_{b}$ is continuous there exists a neighbourhood $N$ of $\alpha_{2}(\eta)$ in $X_{b}$ such that $\alpha_{1}(\xi) N \subset U$. Then there exists a neighbourhood $V \subset W$ of $b$ and a neighbourhood $N^{\prime} \supset K_{b}$ of $\eta$ in $K_{V}$ such that $\alpha_{2}\left(\bar{N}^{\prime} \cap K_{V}\right) \subset U$. Then $\alpha_{1}^{-1}\left(\bar{N}^{\prime} \cap K_{V}, U ; V\right)$ is a neighbourhood of $\xi$ and $N$ is a neighbourhood of $\eta$ such that

$$
\alpha_{1} \alpha_{1}^{-1}\left(\bar{N}^{\prime} \cap K_{V}, U ; V\right) \times_{V} \alpha_{2} N \subset U .
$$

Therefore $e \circ\left(\alpha_{1} \times \alpha_{2}\right)$ is continuous on $K_{V} \times K_{V}$, as required. We deduce

Proposition (5.2). Let $X$ and $Y$ be fibrewise compactly-generated spaces over $B$. Then the fibrewise evaluation function

$$
e: \operatorname{map}_{B}^{\prime}(X, Y) \times_{B}^{\prime} X \rightarrow Y
$$

is continuous.

Lemma (5.3). Let $X$ and $Y$ be fibrewise spaces over $B$, with $X$ fibrewise compactly-generated and $Y$ fibrewise weak Hausdorff. Then

$$
k_{B} \operatorname{map}_{B}\left(X, k_{B} Y\right)=k_{B} \operatorname{map}_{B}(X, Y) .
$$

To make sense, of course, we first have to show that $\operatorname{map}_{B}(X, Y)$ and $\operatorname{map}_{B}\left(X, k_{B} Y\right)$ coincide as fibrewise sets. Now

$$
u_{*}: \operatorname{map}_{B}\left(X, k_{B} Y\right) \rightarrow \operatorname{map}_{B}(X, Y)
$$

is defined, as a fibrewise map, where $u: k_{B} Y \rightarrow Y$ is the identity. On the other hand if $\phi: X_{b} \rightarrow Y_{b}$ is a map, where $b \in B$, then $k \phi: k X_{b} \rightarrow k Y_{b}$ is continuous, and $k X_{b}=X_{b}$ since $X$ is fibrewise compactly-generated. 
So let $\alpha^{\prime}: K \rightarrow \operatorname{map}_{W}\left(X_{W}, k_{W} Y_{W}\right)$ be a fibrewise function, where $W$ is open in $B$ and $K$ is fibrewise compact over $W$. Suppose that

$$
\alpha=u_{*} \alpha^{\prime}: K \rightarrow \operatorname{map}_{W}\left(X_{W}, Y_{W}\right)
$$

is continuous. I assert that $\alpha^{\prime}$ is continuous. For consider a fibrewise compact-open subset $\left(K_{0}, \alpha_{0}, U_{0} ; W_{0}\right)$, where $W_{0}$ is open in $W, U_{0}$ is open in $Y_{W_{0}}$ and $\alpha: K_{0} \rightarrow X_{W_{0}}$ is a fibrewise map. By the previous lemma each point $b$ of $W_{0}$ admits a neighbourhood $V_{0} \subset W_{0}$ such that

$$
e \circ\left(\alpha \times \alpha_{0}\right): K \times{ }_{V} K_{0} \rightarrow Y_{W}
$$

is continuous, and hence $\left(\alpha \times \alpha_{0}\right)^{-1} e^{-1} U_{0}$ is open in $K \times_{V} K_{0}$. Let $\phi_{0}: X_{b} \rightarrow Y_{b}$ be a point of $\left(K_{0}, \alpha_{0}, U_{0} ; W_{0}\right)$. Then $\phi_{0} \alpha_{0} K_{b} \subset U_{b}$ and so there exists a neighbourhood $N$ of $K_{b}$ in $K_{V}$ such that $e\left(\alpha \times \alpha_{0}\right) N \subset U_{0}$. Therefore $\alpha^{\prime}$ is continuous, as asserted, and the proof of (5.3) is complete.

Proposition (5.4). Let $X, Y$ and $Z$ be fibrewise compactly-generated spaces over $B$, with $X$ locally sliceable. Then

$$
\left(\pi_{1 *}, \pi_{2 *}\right): \operatorname{map}_{B}^{\prime}\left(X, Y \times_{B}^{\prime} Z\right) \rightarrow \operatorname{map}_{B}^{\prime}(X, Y) \times_{B}^{\prime} \operatorname{map}_{B}^{\prime}(X, Z)
$$

is an equivalence of fibrewise spaces.

The cartesian property implies that $\left(\pi_{1 *}, \pi_{2 *}\right)$ is injective. To establish surjectivity, let $\phi: X_{b} \rightarrow Y_{b}, \psi: X_{b} \rightarrow Z_{b}$ be continuous, where $b \in B$. Then $\theta=(\phi, \psi)$ : $X_{b} \rightarrow Y_{b} \times Z_{b}$ is continuous, hence $k \circ \theta: X_{b} \rightarrow Y_{b} \times Z_{b}$ is continuous. Therefore $\left(\pi_{1 *}, \pi_{2 *}\right)$ is surjective and hence bijective.

Next we establish the equivalence of the fibrewise compact-open topologies

$$
\operatorname{map}_{B}\left(X, Y \times_{B} Z\right)=\operatorname{map}_{B}(X, Y) \times_{B} \operatorname{map}_{B}(X, Z) .
$$

Consider a fibrewise sub-basic open set on the right, of the form

$$
(K, \alpha, U ; W) \times_{W}(L, \beta, V ; W),
$$

where $W$ is open in $B, U$ is open in $Y_{W}, V$ is open in $Z_{W}, K$ and $L$ are fibrewise compact over $W$ and $\alpha: K \rightarrow X_{W}, \beta: L \rightarrow X_{W}$ are fibrewise maps. This corresponds precisely to the open set

$$
\left(K, \alpha, U \times_{W} Z_{W} ; W\right) \times_{W}\left(L, \beta, Y_{W} \times V ; W\right)
$$

on the left. In the other direction a fibrewise compact-open subset on the left of the special form

$$
\left(K, \alpha, U \times_{W} V ; W\right)
$$

corresponds precisely to the open set

$$
(K, \alpha, U ; W) \times_{W}(K, \alpha, V ; W)
$$

on the right. 
Now consider the case of the general fibrewise compact-open subset $(K, \alpha$, $N ; W$ ) on the left, where $W$ is open in $B, N$ is open in $Y_{W} \times_{W} Z_{W}, K$ is a fibrewise compact over $W$ and $\alpha: K \rightarrow X_{W}$ is a fibrewise map. Let $\phi: X_{b} \rightarrow$ $Y_{b} \times Z_{b}$, where $b \in B$, be a point of $(K, \alpha, N ; W)$, so that $\phi \alpha K_{b} \subset N_{b}$. By (3.22) and (3.26) of [6] $W$ contains a neighbourhood $W_{0}$ of $b$ such that there exist closed subsets $K_{j}$ of $K_{W_{0}}$, open sets $U_{j}$ of $Y_{W_{0}}$ and open sets $V_{j}$ of $Z_{W_{0}}(j=1, \ldots, r)$ with $K_{1} \cup \cdots \cup K_{r}=K_{W_{0}}$ and $U_{j} \times_{W_{0}} V_{j} \subset N_{W_{0}}$ for each index $j$, and with $\phi \alpha K_{j} \subset$ $U_{j} \times_{W_{0}} V_{j}$ for $j=1, \ldots, r$. Then if $\alpha_{j}=\alpha \mid K_{j}$ we have

$$
\phi \in \bigcap_{j=1}^{r}\left(K_{j}, \alpha_{j}, U_{j} \times_{W_{0}} V_{j} ; W_{0}\right) \subset(K, \alpha, N ; W) .
$$

Since each $\left(K_{j}, \alpha_{j}, U_{j} \times_{W_{0}} V_{j} ; W_{0}\right)$ is open in the topology on the right so also is the intersection. Hence $(K, \alpha, N ; W)$ is open in the topology on the right. This proves (5.5).

We now apply $k_{B}$ to both sides of (5.5). By (5.3) the left side becomes

$$
\begin{aligned}
k_{B} \operatorname{map}_{B}\left(X, Y \times_{B} Z\right) & =k_{B} \operatorname{map}_{B}\left(X, k_{B}\left(Y \times_{B} Z\right)\right) \\
& =\operatorname{map}_{B}^{\prime}\left(X, Y^{\prime} \times_{B} Z^{\prime}\right),
\end{aligned}
$$

while the right side, also by (5.3), becomes

$$
\begin{aligned}
k_{B}\left(\operatorname{map}_{B}(X, Y) \times_{B} \operatorname{map}_{B}(X, Z)\right) & =k_{B}\left(\operatorname{map}_{B}(X, Y)\right) \times_{B} k_{B}\left(\operatorname{map}_{B}(X, Z)\right) \\
& =\operatorname{map}_{B}^{\prime}(X, Y) \times_{B}^{\prime} \operatorname{map}_{B}^{\prime}(X, Z) .
\end{aligned}
$$

This completes the proof of (5.4).

We are now ready to establish the exponential law, for our theory, in the following form

Proposition (5.6). Let $X, Y$ and $Z$ be fibrewise compactly-generated spaces over $B$, with $X$ and $Y$ locally sliceable. Then the fibrewise function

$$
\operatorname{map}_{B}^{\prime}\left(X \times_{B}^{\prime} Y, Z\right) \rightarrow \operatorname{map}_{B}^{\prime}\left(X, \operatorname{map}_{B}^{\prime}(Y, Z)\right),
$$

defined by taking right adjoints, is an equivalence of fibrewise spaces.

The first step is to prove

Lemma (5.7). Let $X, Y$ and $Z$ be fibrewise compactly-generated spaces over $B$. Then the right adjoint of a fibrewise map $h: X \times{ }_{B}^{\prime} Y \rightarrow Z$ is a fibrewise map $k: X \rightarrow \operatorname{map}_{B}(Y, Z)$.

For consider a fibrewise compact-open subset $(K, \alpha, V ; W)$ of $\operatorname{map}_{B}(Y, Z)$, where $b$ is a point of $B, W$ is a neighbourhood of $b, V \subset Z_{W}$ is open, $K$ is fibrewise compact over $W$, and $\alpha: K \rightarrow Y_{W}$ is a fibrewise map. Let $k(x) \in$ $(K, \alpha, V ; W)$, where $x \in X_{b}$, in other words $h\left(\{x\} \times \alpha K_{b}\right) \subset V_{b}$. The first projec- 
tion $X_{W} \times_{W} K \rightarrow X_{W}$ is closed, since $K$ is fibrewise compact over $W$. Also $h^{-1} V$ is fibrewise-compactly-open in $X \times_{B} Y$ (i.e. the complement is fibrewisecompactly-closed) and so there exists a neighbourhood $N$ of $x$ in $X_{W}$ such that $N \times_{W} \alpha K \subset h^{-1} V$. Then $N \subset k^{-1}(K, \alpha, V ; W)$ and (5.7) follows.

To deduce (5.6) from this, consider the fibrewise function

$$
\mu: \operatorname{map}_{B}\left(X \times_{B}^{\prime} Y, Z\right) \rightarrow \operatorname{map}_{B}\left(X, \operatorname{map}_{B}(Y, Z)\right),
$$

defined by taking right adjoints. I assert that $\mu$ is an equivalence. Assuming this it only remains to apply the retraction functor $k_{B}$ to both sides. The domain of $\mu$ becomes $\operatorname{map}_{B}^{\prime}\left(X \times_{B}^{\prime} Y, Z\right)$, by definition, while the codomain becomes

$$
\operatorname{map}_{B}^{\prime}\left(X, \operatorname{map}_{B}(Y, Z)\right)=\operatorname{map}_{B}^{\prime}\left(X, \operatorname{map}_{B}^{\prime}(Y, Z)\right),
$$

by (5.3). Hence (5.6) will follow as soon as we have proved the assertion.

We start with the continuity of the fibrewise evaluation function, rearranged as

$$
e: X \times_{B}^{\prime} \operatorname{map}_{B}\left(X \times_{B}^{\prime} Y, Z\right) \times_{B}^{\prime} Y \rightarrow Z .
$$

By (5.7) with $X$ replaced by $X \times_{B}^{\prime} \operatorname{map}_{B}\left(X \times_{B}^{\prime} Y, Z\right)$ we obtain that the right adjoint

$$
X \times_{B}^{\prime} \operatorname{map}_{B}\left(X \times{ }_{B}^{\prime} Y, Z\right) \rightarrow \operatorname{map}_{B}(Y, Z)
$$

is continuous. Switching factors and applying (5.7) again, with $X$ replaced by $\operatorname{map}_{B}\left(X \times{ }_{B}^{\prime} Y, Z\right), Y$ by $X$, and $Z$ by $\operatorname{map}_{B}(Y, Z)$, we find that the right adjoint

$$
\mu: \operatorname{map}_{B}\left(X \times_{B}^{\prime} Y, Z\right) \rightarrow \operatorname{map}_{B}\left(X, \operatorname{map}_{B}(Y, Z)\right)
$$

is continuous as asserted.

To prove that $\mu$ has a continuous inverse, consider the fibrewise evaluation functions

$$
\begin{gathered}
e: \operatorname{map}_{B}(Y, Z) \times_{B}^{\prime} Y \rightarrow Z \\
e: \operatorname{map}_{B}\left(X, \operatorname{map}_{B}(Y, Z)\right) \times_{B}^{\prime} X \rightarrow \operatorname{map}_{B}(Y, Z) .
\end{gathered}
$$

Since the fibrewise spaces are fibrewise compactly-generated the composition

$$
e \circ(e \times i d): \operatorname{map}_{B}\left(X, \operatorname{map}_{B}(Y, Z)\right) \times \times_{B}^{\prime} X \times_{B}^{\prime} Y \rightarrow Z
$$

is continuous. Using the lemma again, with $X$ replaced by $\operatorname{map}_{B}\left(X, \operatorname{map}_{B}(Y, Z)\right)$, $Y$ by $X \times{ }_{B} Y$ and $Z$ by $Z$, we obtain that the right adjoint

$$
\operatorname{map}_{B}\left(X, \operatorname{map}_{B}(Y, Z)\right) \rightarrow \operatorname{map}_{B}\left(X \times_{B}^{\prime} Y, Z\right)
$$


of the composition is defined and continuous. But this is just the inverse of $\mu$, and so the proof of the assertion is complete.

Proposition (5.8). Let $X, Y$ and $Z$ be fibrewise compactly-generated over $B$, with $X$ and $Y$ locally sliceable. Then the fibrewise composition function

$$
\operatorname{map}_{B}^{\prime}(Y, Z) \times_{B}^{\prime} \operatorname{map}_{B}^{\prime}(X, Y) \rightarrow \operatorname{map}_{B}^{\prime}(X, Z)
$$

is continuous.

For as we have seen the fibrewise evaluation functions

$$
e: \operatorname{map}_{B}^{\prime}(Y, Z) \times_{B}^{\prime} Y \rightarrow Z, \quad e: \operatorname{map}_{B}^{\prime}(X, Y) \times_{B}^{\prime} X \rightarrow Y,
$$

are continuous, hence the composition

$$
e \circ(i d \times e): \operatorname{map}_{B}^{\prime}(Y, Z) \times_{B}^{\prime} \operatorname{map}_{B}^{\prime}(X, Y) \times_{B}^{\prime} X \rightarrow Z
$$

is continuous. Applying (5.7), with $X$ replaced by $\operatorname{map}_{B}^{\prime}(Y, Z) \times_{B}^{\prime} \operatorname{map}_{B}^{\prime}(X, Y)$, $Y$ by $\mathrm{X}$ and $Z$ by $Z$, and with $h$ replaced by $e \circ(i d \times e)$, we obtain that the right adjoint

$$
\operatorname{map}_{B}^{\prime}(Y, Z) \times_{B}^{\prime} \operatorname{map}_{B}^{\prime}(X, Y) \rightarrow \operatorname{map}_{B}(X, Z)
$$

of the composition is continuous. Now apply the retraction functor $k_{B}$ and the conclusion follows.

\section{§6. Fibrewise Pointed Mapping Spaces}

Similar results to these hold in the fibrewise pointed theory, as follows. Let $X$ and $Y$ be fibrewise pointed spaces over $B$. As in [6] we denote by $\operatorname{map}_{B}^{B}(X, Y)$ the subspace of $\operatorname{map}_{B}(X, Y)$ consisting of pointed maps of the fibres. When $X$ is locally sliceable and $Y$ is fibrewise weak Hausdorff we write

$$
\operatorname{map}_{B}^{\prime B}(X, Y)=k_{B} \operatorname{map}_{B}^{B}(X, Y) .
$$

Equivalently we regard $\operatorname{map}_{B}^{\prime B}(X, Y)$ as the subspace of $\operatorname{map}_{B}^{\prime}(X, Y)$ consisting of pointed maps of the fibres. From (9.24) of [6] and (5.8) above we obtain

Proposition (6.1). Let $X$ and $Y$ be fibrewise compactly-generated fibrewise pointed spaces over $B$, with $X$ locally sliceable. Then the fibrewise evaluation function

$$
\operatorname{map}_{B}^{\prime B}(X, Y) \wedge_{B}^{\prime} X \rightarrow Y
$$

is continuous.

More generally, from (9.23) of [6] and (5.8) above we obtain 
Proposition (6.2)。 Let $X, Y$ and $Z$ be fibrewise compactly-generated fibrewise pointed spaces over $B$, with $X$ and $Y$ locally sliceable. Then the fibrewise composition function

$$
\operatorname{map}_{B}^{\prime B}(Y, Z) \wedge_{B}^{\prime} \operatorname{map}_{B}^{\prime B}(X, Y) \rightarrow \operatorname{map}_{B}^{\prime B}(X, Z)
$$

is continuous.

Our aim, of course, is to establish the fibrewise compactly-generated version of the exponential law, for fibrewise pointed mapping-spaces. For this another technical result is required, as follows. Let $X$ be fibrewise compactlygenerated over $B$. Let $A$ be a closed subspace of $X$ such that the fibrewise pointed space $X /{ }_{B} A$ obtained from $X$ by fibrewise collapsing $A$ is fibrewise weak Hausdorff. By precomposition with the fibrewise quotient map

$$
\pi:(X, A) \rightarrow\left(X /{ }_{B} A, B\right)
$$

we obtain a fibrewise map

$$
\pi^{*}: \operatorname{map}_{B}^{B}\left(X /{ }_{B} A, Y\right) \rightarrow \operatorname{map}_{B}((X, A),(Y, B)),
$$

for each fibrewise pointed space $Y$. Here the codomain means the subspace of $\operatorname{map}_{B}(X, Y)$ consisting of maps $\phi: X_{b} \rightarrow Y_{b}(b \in B)$ such that $\phi_{b} A_{b}=t(b)$, where $t$ is the section of $Y$. Clearly $\pi^{*}$ is bijective. I assert that after applying the functor $k_{B}, \pi^{*}$ yields a natural equivalence between $\left.\operatorname{map}_{B}^{\prime B}(X)_{B} A, Y\right)$ and $\operatorname{map}_{B}^{\prime}((X, A),(Y, B))$, as fibrewise pointed spaces.

For let $\alpha: K \rightarrow \operatorname{map}_{W}^{W}\left(X_{W} /{ }_{W} A_{W}, Y_{W}\right)$ be a fibrewise function, where $W$ is open in $B$ and $K$ is fibrewise compact over $W$. Suppose that $\beta=\pi^{*} \alpha: K \rightarrow$ $\operatorname{map}_{W}\left(\left(X_{W}, A_{W}\right),\left(Y_{W}, \mathrm{~W}\right)\right)$ is continuous. I assert that $\alpha$ is continuous. For let $\xi \in K_{b}$, where $b \in B$, and let $(L, \gamma, U ; W)$ be a fibrewise compact-open neighbourhood of $\alpha(\xi)$, where $U$ is open in $Y_{W}, L$ is fibrewise compact over $W$ and $\gamma: L \rightarrow X_{W} /{ }_{W} A_{W}$ is a fibrewise map. We distinguish two cases, of which the first is straightforward.

Suppose that $\gamma L$ does not meet $s W$, where $s$ is the natural section of $X /{ }_{B} A$. Then $\gamma=\pi \circ \delta$, where $\delta: L \rightarrow X_{W}$ is a fibrewise map, and $\beta^{-1}(L, \delta, U ; W)$ is a neighbourhood of $\xi$ such that

$$
\alpha \beta^{-1}(L, \delta, U ; W) \subset(L, \gamma, U ; W) .
$$

Suppose, however, that $\gamma L$ meets $s W$ in $s W_{0}$, say, where $W_{0}$ is necessarily closed in $W$. By (5.1) the fibrewise function

$$
e \circ(\alpha \times i d): K_{V} \times_{V} X_{V} \rightarrow Y_{V}
$$

is continuous, for some neighbourhood $V \subset W$ of $b$, and so the fibrewise function

$$
e \circ(\beta \times i d): K_{V} \times{ }_{V}\left(X_{V} / A_{V}\right) \rightarrow Y_{V}
$$


is continuous, since $K_{V}$ is fibrewise compact over $V$. Hence $(\beta \times i d)^{-1} e^{-1} U$ is a neighbourhood of $\beta^{-1} s W_{0} \times_{W_{0}} s W_{0}$ in $K_{V} \times_{V} X_{V}$ and so there exists a neighbourhood $M$ of $\beta^{-1} s W_{0}$ in $K_{V}$ and a neighbourhood $N$ of $s W_{0}$ in $X_{V} / A_{V}$ such that

$$
M \times{ }_{V} N \subset(\beta \times i d)^{-1} e^{-1} U .
$$

Set $L^{*}=L-L \cap \gamma^{-1} N$; then $L^{*}$ is fibrewise compact over $V$ and does not meet $s W$. It follows that

$$
\beta^{-1} M \cap \alpha^{-1}\left(L, \gamma^{*}, U ; V\right)
$$

is a neighbourhood of $\xi$ in $K$, where $\gamma^{*}=\pi^{-1} \gamma \mid L_{V}^{*}$, and any $\eta$ in this neighbourhood will be such that $\beta(\eta)\left(\pi^{-1} N\right) \subset U$ and $\beta(\eta)\left(L_{V}^{*}\right) \subset U$. Since $L \subset L^{*} U$ $\pi^{-1} N$ it follows that $\pi^{*} \alpha(\eta) \in(L, \delta, U ; W)$, as required.

This completes the proof of the technical result, from which we deduce our exponential law as follows.

Proposition (6.3). Let $X, Y$ and $Z$ be fibrewise compactly-generated fibrewise pointed spaces over $B$. Then the fibrewise pointed spaces $\operatorname{map}_{B}^{\prime B}\left(X \wedge_{B}^{\prime} Y, Z\right)$ and $\operatorname{map}_{B}^{\prime B}\left(X, \operatorname{map}_{B}^{\prime B}(Y, Z)\right)$ are naturally equivalent.

To see this, let us replace $Y$ by $Z$ and $(X, A)$ by $\left(X \times_{B} Y, X \vee_{B} Y\right)$, in the technical result. Then we obtain a natural equivalence between $\operatorname{map}_{B}^{\prime B}\left(X \wedge_{B}^{\prime} Y\right)$ and the subspace

$$
\operatorname{map}_{B}^{\prime}\left(\left(X \times_{B} Y, X \vee_{B} Y\right) ;(Z, B)\right)
$$

of $\operatorname{map}_{B}^{\prime}\left(X \times_{B} Y, Z\right)$. As we have seen in (5.6), the latter is equivalent to $\operatorname{map}_{B}^{\prime}\left(X, \operatorname{map}_{B}^{\prime}(Y, Z)\right)$. In the equivalence the subspace corresponds precisely to $\operatorname{map}_{B}^{\prime B}\left(X, \operatorname{map}_{B}^{\prime B}(Y, Z)\right)$. This completes the proof.

\section{References}

[1] Booth, P. I., The section problem and the lifting problem, Math. Zeit., 121 (1971), $273-87$.

[2] Booth, P. I. and Brown, R., On the application of fibred mapping spaces to exponential laws for bundles, ex-spaces and other categories of maps, Gen. Topology and its Appl., 8 (1978), 165-79.

[3] - Spaces of partial maps, fibred mapping spaces and the compact-open topology, Gen. Topology and its Appl., 8 (1978), 181-95.

[4] Brown, R., Function spaces and product topologies, Quart. J. Math. Oxford (2), 15 (1964), 238-50.

[5] James, I. M., General Topology and Homotopy Theory, Springer Verlag, 1985.

[6] - Fibrewise Topology, Cambridge Univ. Press, 1990.

[7] McCord, M. C., Classifying spaces and infinite symmetric products, Trans. Amer. Math. Soc., 146 (1969), 273-98.

[8] Min, K. C. and Lee, S. J., Fibrewise convergence and exponential laws, Tsukuba J. Math., 16 (1992), 53-62.

[9] Steenrod, N. E., A convenient category of topological spaces, Michigan Math. J., 14 (1967), 133-52. 
